\title{
Multiphoton Ionization and Fragmentation of Hydrogen Chloride: A Diatomic Still Good for a Surprise
}

\author{
Christof Maul, ${ }^{1}$ Alexey I. Chichinin, ${ }^{1,2}$ and Karl-Heinz Gericke ${ }^{1}$ \\ ${ }^{1}$ Institut für Physikalische und Theoretische Chemie, Technische Universität Braunschweig, 38106 Braunschweig, Germany \\ ${ }^{2}$ Institute of Chemical Kinetics and Combustion, Russian Academy of Sciences, Novosibirsk 630090, Russia
}

Correspondence should be addressed to Christof Maul, c.maul@tu-braunschweig.de

Received 1 October 2010; Revised 14 February 2011; Accepted 3 March 2011

Academic Editor: B. A. Malomed

Copyright (C) 2011 Christof Maul et al. This is an open access article distributed under the Creative Commons Attribution License, which permits unrestricted use, distribution, and reproduction in any medium, provided the original work is properly cited.

\begin{abstract}
The competition between multiphoton ionization and fragmentation in the diatomic molecule hydrogen chloride is reviewed. Emphasis is laid on recent experimental results employing chemical imaging methods in order to obtain kinetic energy distributions and angular distributions of photoproducts. The energy range considered is 15 to $20 \mathrm{eV}$, equivalent to the absorption of three or four photons in the ultraviolet wavelength range. The role of Rydberg states as resonantly excited intermediate states in the ionization/fragmentation processes is assessed. Mixing among ${ }^{1} \Sigma^{+}$states gives rise to peculiarly shaped double minimum potential energy curves which allow for the production of hydrogen and chlorine atomic and ionic fragments via several competing pathways, in addition to the production of molecular $\mathrm{HCl}^{+}$ions. States with different electronic properties show a qualitatively different behaviour from $\Sigma^{+}$states. Accidental resonances between states of differing orbital angular momentum or multiplicity serve to override these differences and cause subtle as well as significant deviations from the unperturbed behaviour.
\end{abstract}

\section{Introduction}

Hydrogen halides are molecules of fundamental interest in chemistry and physics and have extensively been studied theoretically as well as experimentally. In many respects, they can be regarded as prototypes of heteronuclear diatomic molecules. While the electronic ground states of the hydrogen halides are in general well understood, their Rydberg and valence electronic structure still bears some surprises for the experimentalist and for the theoretician. For example, competing multiphoton dissociation and ionization processes are observed as a consequence of Rydberg-valence interactions at large internuclear distances, a behaviour which is primarily associated with ${ }^{1} \Sigma^{+}\left(0^{+}\right)$and ${ }^{3} \Sigma^{-}\left(0^{+}\right)$intermediate states.

The focus of the work summarized here lies in the investigation of the photoionization of $\mathrm{HCl}$ following two-photon excitation of its Rydberg states. For $\mathrm{HBr}$ [1-3], and to a lesser extent for HI [4], similar studies and similar conclusions have been reported, whereas much less is known about HF, most probably due to the difficulty of state-selective fluorine atom detection [5] and to the more problematic chemical properties of HF making gas handling significantly more demanding.

Three main issues are associated with resonance enhanced multiphoton ionization of $\mathrm{HCl}$. First, if the $B^{1} \Sigma^{+}\left(0^{+}\right)$state is accessed as resonantly intermediate state, its peculiar double-well structure opens the door for subsequent photoabsorption at very large internuclear distances, giving rise to complex competition between ionization and fragmentation schemes. Second, specific spatially anisotropic photoproduct distributions result from the electronic nature and symmetry properties of the involved "virtual" states in the two-photon resonant excitation and thus help to gain an understanding of the complex ionization-fragmentation processes. Last, resonances of individual rovibrational levels of the $B^{1} \Sigma^{+}\left(0^{+}\right)$state with rovibrational levels of ${ }^{2 S+1} \Lambda$ states with $S$ and $\Lambda$ greater than 0 mix the nonstandard $B$ state behaviour into ionization patterns of the latter.

All three phenomena are ideally studied by photoion or photoelectron imaging techniques. Photoion imaging allows one to simultaneously determine kinetic energy as well as state-selected spatial distributions of ionized photoproducts. 
These techniques include the two-dimensional (2D) imaging technique [6], its two-dimensional velocity mapping counterpart (2D-VMI) $[7,8]$, and the corresponding threedimensional (3D) techniques [9-11]. Here, special emphasis will be laid on experiments performed using the threedimensional velocity map imaging technique developed at Braunschweig (3D-VMI) [10, 11]. A comprehensive review of 3D-VMI has recently been given in [12].

Almost all recent work published on the ionization/ fragmentation of $\mathrm{HCl}$ relies on the fundamental work of Green et al. who have reported a remarkably detailed and comprehensive survey of the excited electronic states of $\mathrm{HCl}$ in three parts [13-15]. Using resonance enhanced multiphoton ionization $((2+1)$-REMPI) and time-of-flight mass spectrometry (TOFMS), they analyzed over 120 band systems in the 77000 to $96000 \mathrm{~cm}^{-1}$ region. In several band systems, they observed anomalous rotational line intensities and broadened linewidths which are consistent with predissociation.

In continuation of their work, Kvaran and coworkers [16-18] have investigated several of those states with a predissociation signature by a mass sensitive REMPI timeof-flight technique. Branching ratios for parent molecule ionization and fragmentation producing either $\mathrm{H}^{+}$or $\mathrm{Cl}^{+}$ have been studied for both $\mathrm{H}^{35} \mathrm{Cl}$ and $\mathrm{H}^{37} \mathrm{Cl}$ isotopologues on a quantum state selective basis. Special attention was paid to $\Delta(\Lambda=2)$ states in the singlet and triplet manifolds for which accidental resonances with the double-well $B^{1} \Sigma^{+}\left(0^{+}\right)$ state occur.

Photoproduct spatial anisotropies and kinetic energy distributions were analyzed by Loock and coworkers $[1,3,19]$ and by Gericke and co-workers $[20,21]$ using velocity map imaging techniques. Both groups investigated the ionization/fragmentation pathways through various vibrational bands of the $B^{1} \Sigma^{+}\left(0^{+}\right)$state. Very recently, Kauczok et al. [22] have reported similar studies on the singlet and triplet $\Delta$ states that previously were identified as being in resonance with selected rovibrational states of the $B^{1} \Sigma^{+}\left(0^{+}\right)$state [1618].

A number of additional experimental studies have been published on $\mathrm{HCl}$ ionization. Among them are several studies using photoelectron spectroscopy which were conducted primarily to investigate the electronic structure of the $\mathrm{HCl}^{+}$ molecular ion [23-28]. While being helpful for interpreting the results of the photoion experiments mentioned above, these studies are beyond the scope of this work and will not be discussed in detail here.

Theoretical calculations of excited potential energy curves of $\mathrm{HCl}$ are abundant [29-39], however the ones relevant to the case discussed here date back well into the last century, and discrepancies between theoretical and experimental results have not yet fully been removed. The general topology of the relevant potential energy curves is beyond doubt, but understanding the subtle details with respect to the competition between fragmentation and ionization seems to require more accurate potentials than are available today. Fortunately, renewed interest in $\mathrm{HCl}$ excited potential energy surfaces has been raised by the new, intriguing experimental results, and high quality calculations are under way [40].

\section{Potential Energy Curves: A Simplified View of the Essentials}

The ground electronic state of $\mathrm{HCl}$ is $X^{1} \Sigma^{+}\left(0^{+}\right)$, while the ground and first excited electronic state of the molecular ion $\mathrm{HCl}^{+}$are $X^{2} \Pi$ and $A^{2} \Sigma^{+}$, respectively. Figure 1 shows a selection of potential energy curves of $\mathrm{HCl}$. Generally, neutral states are shown by solid lines and ionic states by dashed lines. The ground $X^{1} \Sigma^{+}\left(0^{+}\right)$and excited $B^{1} \Sigma^{+}\left(0^{+}\right)$ states are shown in black, the ionic $X^{2} \Pi$ ground state is shown in red and the ionic $A^{2} \Sigma^{+}$excited state is shown in blue. The same colour coding has been used for the neutral Rydberg states converging either to the $X^{2} \Pi$ or to the $A^{2} \Sigma^{+}$state. Asymptotically, the $X^{2} \Pi$ ground ionic state correlates with neutral ground state atomic hydrogen and a chlorine ion $\left(\mathrm{H}+\mathrm{Cl}^{+}\right)$as opposed to the $A^{2} \Sigma^{+}$state which asymptotically correlates with an $\mathrm{H}^{+}$ion and a neutral ground state chlorine atom $\left(\mathrm{H}^{+}+\mathrm{Cl}\right)$.

Accordingly, red and blue coloured Rydberg states correlate with the corresponding ground and excited neutral atoms $\left(\mathrm{H}+\mathrm{Cl}^{*}\right.$ and $\left.\mathrm{H}^{*}+\mathrm{Cl}\right)$. The Rydberg states shown in Figure 1 were obtained by shifting the corresponding ionic curve by the ionization energy of the excited state $\left(\mathrm{H}^{*}\right.$ or $\mathrm{Cl}^{*}$ ) atom. Only few of the neutral Rydberg states are shown in Figure 1 in order to illustrate the general scheme rather than to obscure it by too many details. For a more complete set of potential energy curves the reader is referred to [20].

Generally, neutral Rydberg states occur as bound and as repulsive states, just like the corresponding ionic states. In Figure 1, the "full" set of bound and repulsive Rydberg states is only shown for the "blue" curves correlating to $\mathrm{H}^{*}(n=2)$ $+\mathrm{Cl}$, while the bound state is omitted for the "red" Rydberg states correlating to $\mathrm{H}+\mathrm{Cl}^{*}$. One of those bound Rydberg states is responsible for the inner potential well of the $B$ state (i.e., the $E^{1} \Sigma^{+}$state in 0 th order approximation) [29]. The outer potential well of the $B$ state (the $V^{1} \Sigma^{+}$state in 0th order approximation) is due to the ion-pair curve correlating to $\mathrm{H}^{+}$ $+\mathrm{Cl}^{-}$products [29]. Here, for larger distances the interaction is mainly Coulombic giving rise to the energy minimum at rather large internuclear distances between 2 and $3 \AA$. It is important to note that the energy of the asymptotic products $\mathrm{H}^{+}+\mathrm{Cl}^{-}$lies above the energy of the $\mathrm{H}+\mathrm{Cl}^{*}(4 \mathrm{~s})$ system (red solid curve), but below the energy of the $\mathrm{H}^{*}(n=2)$ $+\mathrm{Cl}$ system (blue solid curve). It goes without saying that among the omitted bound (red) Rydberg states are several that are characterized by an orbital angular momentum $\Lambda>$ 0 , in particular the $F^{1} \Delta$ and $f^{3} \Delta$ states that were shown by Kvaran and coworkers for individual rovibrational levels to be in resonance with rovibrational levels of the $B^{1} \Sigma^{+}$state $[16,18] . F^{1} \Delta$ and $f^{3} \Delta$ states are energetically very close to $E^{1} \Sigma^{+}$, thus fine-tuning the excitation energy in this narrow energy range allows the experimentalist to access states with differing electronic. This situation is a very fortunate one in order to investigate the influence of electronic properties unobscured by dominating effects of a change in energy of the studied system. 


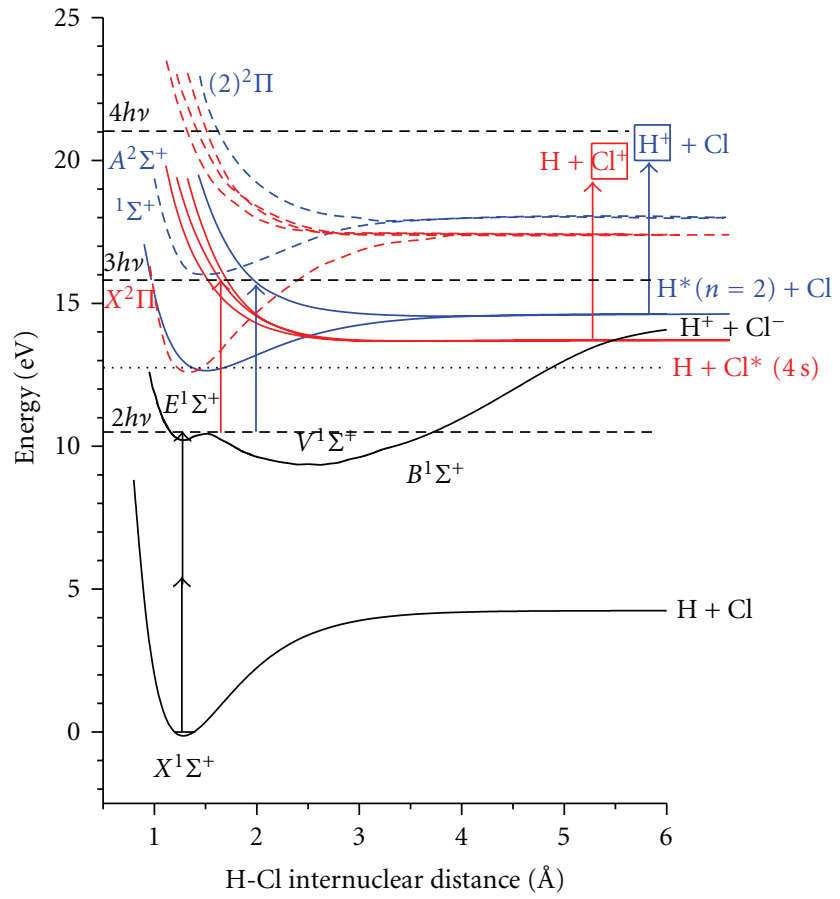

Figure 1: Potential energy curves of $\mathrm{HCl}$. Solid black curves: initial and final state of the $B\left({ }^{1} \Sigma^{+}\right) \leftarrow X\left({ }^{1} \Sigma^{+}\right)$two-photon transition. Dashed curves: ionic states correlating to $\mathrm{H}+\mathrm{Cl}^{+}$(red) and $\mathrm{H}^{+}+$ $\mathrm{Cl}$ (blue). Solid coloured curves: neutral Rydberg states converging to $\mathrm{H}+\mathrm{Cl}^{+}$(red) and $\mathrm{H}^{+}+\mathrm{Cl}$ (blue). Red arrows illustrate the pathway for producing $\mathrm{Cl}^{+}$ions, blue arrows illustrate one of several pathways that produce $\mathrm{H}^{+}$ions. Dashed and dotted black straight lines denote the 2, 3, and 4 photon energy levels and the ionization limit, respectively. The asymptotic value for the $\mathrm{H}^{+}+\mathrm{Cl}^{-}$ion pair remains between $\mathrm{H}^{*}(n=2)+\mathrm{Cl}$ and $\mathrm{H}+\mathrm{Cl}^{*}(4 \mathrm{~s})$.

\section{Ionization and Fragmentation through the Double Well $B\left({ }^{1} \Sigma^{+}\right)$State}

Figure 1 also illustrates the general multiphoton excitation scheme. A selected rovibrational level $\left(v^{\prime \prime}, J^{\prime \prime}\right)$ of the $X^{1} \Sigma^{+}$ state is resonantly excited by two-photon absorption to a rovibrational level $\left(v^{\prime}, J^{\prime}\right)$ of an intermediate state, for example, the inner $E$ well of the $B^{1} \Sigma^{+}$state in Figure 1 . Absorption of a third photon results in a superexcited $\mathrm{HCl}^{* *}$ molecule which will subsequently either undergo fragmentation or autoionize into a stable $\mathrm{HCl}^{+}$ion characterized by quantum numbers $\left(v^{+}, J^{+}\right)$. The molecular ion and the ionpair fragments $\mathrm{H}^{+}+\mathrm{Cl}^{-}$are observable at the three-photon level, while in all other cases, absorption of a fourth photon will yield the observed photoproducts $\mathrm{H}^{+}$or $\mathrm{Cl}^{+}$.

Green et al. [13-15] have reported a long $\left(v^{\prime}=3\right.$ to 32$)$ vibrational progression of the double well $B[V / E]\left({ }^{1} \Sigma^{+}\right)$ Rydberg/ion-pair state. $V$ and $E$ are commonly used to refer to (sub)states rather located in the outer (ion-pair) or the inner (Rydberg) well of the double-well $B$ potential curve [29].

Figure 2 shows ion yields of the $\mathrm{HCl}^{+}$parent ion (observed at mass 36) and of the $\mathrm{Cl}^{+}$daughter fragment (observed at mass 35) for four vibrational bands belonging

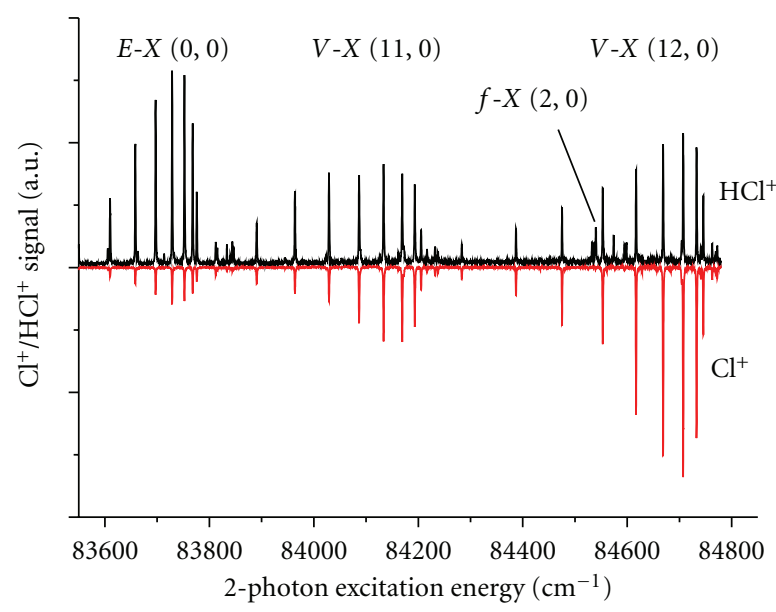

FIgURE 2: Four vibrational bands of the $B[V / E]\left({ }^{1} \Sigma^{+}\right) \leftarrow X\left({ }^{1} \Sigma^{+}\right)$ and the $f\left({ }^{3} \Delta_{2}\right) \leftarrow X\left({ }^{1} \Sigma^{+}\right)$two-photon transitions. The upper trace was monitored on mass 36 of the parent ion $\mathrm{H}^{35} \mathrm{Cl}^{+}$, the lower trace was simultaneously monitored on the $\mathrm{Cl}^{+}$fragment mass 35 . For the $B[V / E]\left({ }^{1} \Sigma^{+}\right) \leftarrow X\left({ }^{1} \Sigma^{+}\right)$bands, the fragmentation ratio does not only rise with excitation energy, but also crucially depends on vibrational and rotational quantum numbers. At the same time, the $f\left({ }^{3} \Delta_{2}\right) \leftarrow X\left({ }^{1} \Sigma^{+}\right)$two-photon transition which is responsible for the weak $\mathrm{HCl}^{+}$lines around $84500 \mathrm{~cm}^{-1}$ does not show any significant fragmentation at all at the employed sensitivity level.

to the electronic transitions $B[V / E]\left({ }^{1} \Sigma^{+}\right) \leftarrow X\left({ }^{1} \Sigma^{+}\right)$and $f\left({ }^{3} \Delta_{2}\right) \leftarrow X\left({ }^{1} \Sigma^{+}\right)$. While not being displayed in Figure 2, a similar picture is obtained for the $\mathrm{H}^{+}$daughter fragment. Two main issues when exciting the double-well $B[V / E]$ state are illustrated in the spectra shown in Figure 2.

The fragmentation ratio $\left(\mathrm{Cl}^{+} / \mathrm{HCl}^{+}\right)$does not only show a smooth dependence on the excitation energy, but also crucially depends on the nature of the electronic states as well as on vibrational and rotational quantum numbers $[16,18,20]$. However, independently of the excitation energy, there exist bands which do not exhibit any significant fragmentation at all. One such band, arising from the $f\left({ }^{3} \Delta_{2}\right) \leftarrow X\left({ }^{1} \Sigma^{+}\right)$transition, is shown in Figure 2 around $84500 \mathrm{~cm}^{-1}$, within the $V-X(12,0)$ band. The reason for this peculiar behaviour is the mixing of the ion-pair $(V)$ and the Rydberg $(E)$ potential energy curves resulting in the double minimum $B$ curve shown in Figure 1. Bound electronic Rydberg states other than ${ }^{1} \Sigma^{+}\left(\right.$or $\left.0^{+}\right)$, but with potential energy curves very similar to the $E$ curve, cannot mix with the ion-pair $V$ state and remain "pure" single-well states at small equilibrium nuclear separation. The latter is the case for the weak lines of the $f\left({ }^{3} \Delta_{2}\right) \leftarrow X\left({ }^{1} \Sigma^{+}\right)$twophoton transition shown in Figure 2.

The double-well character of the $B$ state has far-reaching consequences. By passing over or tunneling through the barrier separating the two minima of the $B$ state stable nuclear configurations can be realized at very large internuclear distances. Absorption of another photon at large internuclear distance by the molecule previously excited into the $B[V / E]$ state can occur via several ionization and fragmentation 
pathways which are not available to $\mathrm{HCl}$ molecules excited to one of the pure Rydberg states confined to the inner well at small internuclear separation. For this reason, the result of additional photon absorption of a $B$ state $\mathrm{HCl}$ molecule is very different from that of a pure Rydberg state $\mathrm{HCl}$ molecule.

The situation can be understood by taking a closer look at the potential energy surfaces of Figure 1. Shown are initial and final states of the $B\left({ }^{1} \Sigma^{+}\right) \leftarrow X\left({ }^{1} \Sigma^{+}\right)$twophoton transition. The small barrier between the inner Rydberg well $E$ and the outer ion-pair well $V$ can either be surpassed, if the excitation energy is sufficient, or tunnelled through, if the energy is slightly below the barrier. In either case the vibrational wavefunction assumes a large value at large internuclear distances. In these cases the whole range of internuclear distances from 2 to $4 \AA$ becomes available for subsequent photon absorption processes. Red and blue arrows in Figure 1 illustrate additional pathways that access repulsive Rydberg states at large internuclear distances converging either to the $\mathrm{H}+\mathrm{Cl}^{+}$or to the $\mathrm{H}^{+}+$ $\mathrm{Cl}$ limit. These repulsive Rydberg states rapidly decay into an excited and a ground state atom, that is, either to $\mathrm{H}+\mathrm{Cl}^{*}(4 \mathrm{~s}$, $4 \mathrm{p}, 3 \mathrm{~d})$ or to $\mathrm{H}^{*}(n=2)+\mathrm{Cl}$. These excited atoms are easily ionized by the radiation field and are detected by the particle detector.

While for $\mathrm{Cl}^{+}$ions the red arrows in Figure 1 show the only way how $\mathrm{Cl}^{+}$can be generated, for $\mathrm{H}^{+}$there exist several possibilities of which only one is depicted in Figure 1 by the blue arrows (path I). Two other pathways are (a) population of a bound superexcited Rydberg state by absorption of a third photon followed by nonadiabatic transition to the ionpair potential curve and decay into $\mathrm{H}^{+}+\mathrm{Cl}^{-}$(path II) and (b) ionization of $\mathrm{HCl}(\mathrm{B})$ by absorption of a third photon followed by photodissociation of $\mathrm{HCl}^{+}$into $\mathrm{H}^{+}+\mathrm{Cl}$ by absorption of a fourth photon (path III).

Close examination of the energetics of the potential energy curves related to paths (I) to (III) shows that the kinetic energy release is different for any of the three dissociation pathways. Hence, observing the kinetic energy distribution of $\mathrm{H}^{+}$fragments in the experiment allows one to unambiguously determine the contribution of each of the three paths to the overall decay process. In addition, if a 3D imaging scheme is used for particle detection, one obtains the spatial fragment distribution "for free" which allows one to identify the symmetry properties of the electronic states which govern the fragmentation process.

Figure 3 shows an example of a two-dimensional meridian projection of a three-dimensional (3D) $\mathrm{H}^{+}$ion image obtained by the $3 \mathrm{D}$ velocity map imaging (3DVMI) technique, in addition to the (one-dimensional) speed distribution. A meridian plot is a projection of a point $\left(v_{x}, v_{y}\right.$, and $v_{z}$ ) into a plane containing the origin, while the absolute speed is left constant [20]. It is important to realize that both projections were obtained from one fully three-dimensional image and that no reconstruction algorithm whatsoever was needed for obtaining the three-dimensional distribution from a set of lower-dimensional measurements as it is the case in time-of-flight experiments or in two-dimensional imaging or velocity mapping schemes.

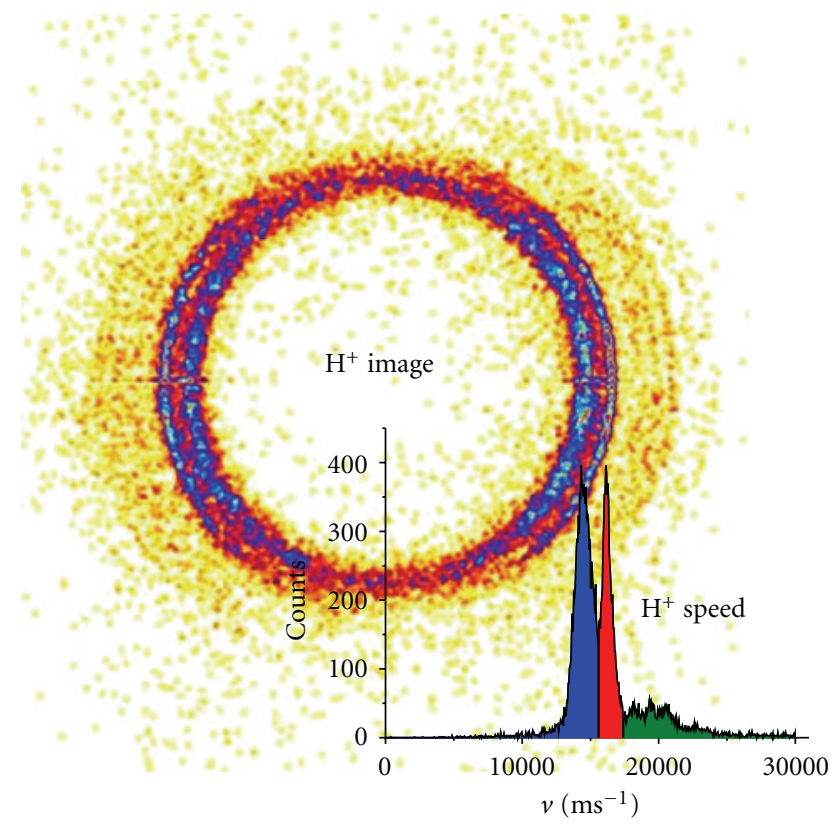

FIGURE 3: Velocity map image of $\mathrm{H}^{+}$ions generated by competing dissociation and ionization processes following two-photon excitation of the $Q(0)$ line of the $B[V]\left({ }^{1} \Sigma^{+}, v^{\prime}=12\right) \leftarrow X\left({ }^{1} \Sigma^{+}, v^{\prime \prime}=\right.$ $0)$ band at $236.000 \mathrm{~nm}$. Shown are the two-dimensional meridian projection and matching one-dimensional speed distribution obtained from the fully three-dimensional (3D) momentum vector distribution of $\mathrm{H}^{+}$ions observed by the $3 \mathrm{D}$ velocity map imaging (3DVMI) technique. The colour coding of the speed distribution assigns the observed $\mathrm{H}^{+}$ions to the three fragmentation pathways discussed in the text (blue/slow: path I, red/medium: path II, green/ fast: path III).

For clarity, in Figure 3 the contributions of the three pathways are colour coded. The slowest "blue" ions correspond to path I, that is, they were generated on a repulsive Rydberg state decaying into an electronically excited $\mathrm{H}^{*}(n=$ 2 ) atom and a ground state chlorine atom. Path I is illustrated in Figure 4. Here, the kinetic energy release in the observed $\mathrm{H}^{*}(n=2)$ fragment is determined by the difference of the three-photon energy level (at the upper end of the blue arrow) and the energy of $\mathrm{H}^{*}(n=2)+\mathrm{Cl}$ (asymptotic value of the blue potential energy curve for large internuclear separation).

The fastest "green" ions are $\mathrm{H}^{+}$ions generated in the photodissociation of ionized $\mathrm{HCl}^{+}$parent ions (path III, see Figure 5). The state being excited upon absorption of the third photon is identical to the one in the "blue" case discussed before and shown in Figure 4. Therefore this state has been termed a "gateway" state. From analysis of the angular anisotropy it has been found to be a ${ }^{3} \Pi_{0}$ state (see Section 5). Now, however, autoionization into vibrationally excited levels of the ionic ground state potential takes place, from where absorption of a fourth photon produces $\mathrm{H}^{+}+$ $\mathrm{Cl}$. The fine structure in the "green" portion at the upper end of the speed distribution consisting of individual peaks around $20000 \mathrm{~m} / \mathrm{s}$ in Figure 3 reflects the distribution of the $\mathrm{HCl}^{+}$parent ions over different vibrational states prior to their dissociation, three of which are shown in Figure 5. 


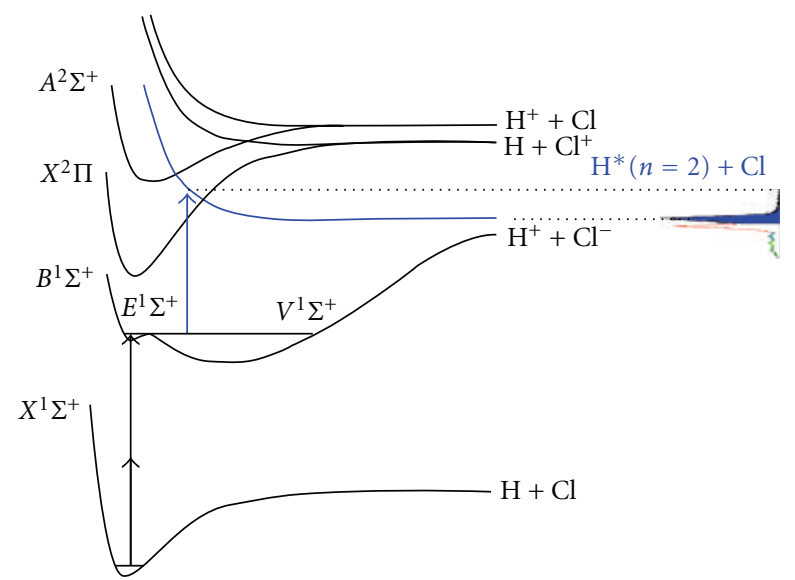

FIgURE 4: Path I: the slowest observed $\mathrm{H}^{+}$ions are produced from the decay of superexcited $\mathrm{HCl}^{* *}$ into $\mathrm{H}^{*}(n=2)+\mathrm{Cl}$, followed by nonresonant ionization of $\mathrm{H}^{*}(n=2)$ by absorption of a fourth photon. The kinetic energy of the observed $\mathrm{H}^{+}$fragment is determined in the third photon absorption step. The colour coding matches that of Figure 3.

Finally, medium fast "red" $\mathrm{H}^{+}$ions are produced via path II (see Figure 6), that is, in conjunction with a $\mathrm{Cl}^{-}$ion. The ion-pair path (II) in particular is subject to subtle details in the topology of the involved potential energy curves. This dependence manifests itself in the intensity oscillations of the ion-pair peak with the vibrational quantum number of the initially excited $B\left({ }^{1} \Sigma^{+}\right)$state as it was observed by Loock and coworkers (cf. [3, Figure 2]).

Figure 7 combines the information about the three individual paths (I), (II), and (III), depicted above in Figures 4 to 6 . All three paths together result in the typical velocity image shown in Figure 3. $\mathrm{H}^{+}$ions resulting from the dissociation of superexcited $\mathrm{HCl}$ into $\mathrm{H}^{*}(n=2)+\mathrm{Cl}$ and those resulting from autoionization followed by dissociation into $\mathrm{H}^{+}+\mathrm{Cl}$, that is, path I and path III, proceed over the same "gateway" state shown in Figures 4 to 7 , which was found to be a ${ }^{3} \Pi_{0}$ repulsive state in the Rydberg series converging to the ionic $A^{2} \Sigma^{+}$state [19].

Relative contributions of the three paths depend crucially on the evolution of the wave packets created on the relevant potential energy surfaces, that is, on their topologies. Velocity Map Imaging provides a valuable tool for exploring the complex behaviour of a seemingly simple molecular system upon multiphoton excitation.

\section{Ionization and Fragmentation through Other Electronic States}

If intermediate states other than ${ }^{1} \Sigma^{+}$are accessed by the resonant two-photon excitation step in REMPI, normally $\mathrm{HCl}^{+}$molecular ions are produced, and no or only negligible fragmentation into either $\mathrm{H}$ or $\mathrm{Cl}$ is observed (cf. [13, Table 1]). A typical example are the weak $\mathrm{HCl}^{+}$lines around $84500 \mathrm{~cm}^{-1}$ which belong to the $f\left({ }^{3} \Delta_{2}\right) \leftarrow X\left({ }^{1} \Sigma^{+}\right)$twophoton transition, as shown in Figure 2.

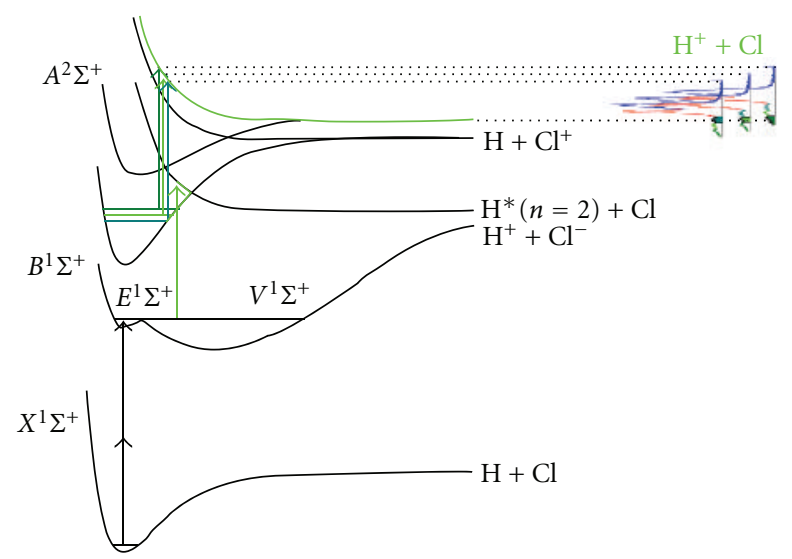

Figure 5: Path III: the fastest observed $\mathrm{H}^{+}$ions are produced from autoionization of superexcited $\mathrm{HCl}^{* *}$ to $\mathrm{HCl}^{+}\left(v^{+}\right)$with $v^{+}>5$, followed by photodissociation of $\mathrm{HCl}^{+}\left(v^{+}\right)$by absorption of a fourth photon. The kinetic energy of the observed $\mathrm{H}^{+}$fragment is determined in the fourth photon absorption step. The colour coding matches that of Figure 3.

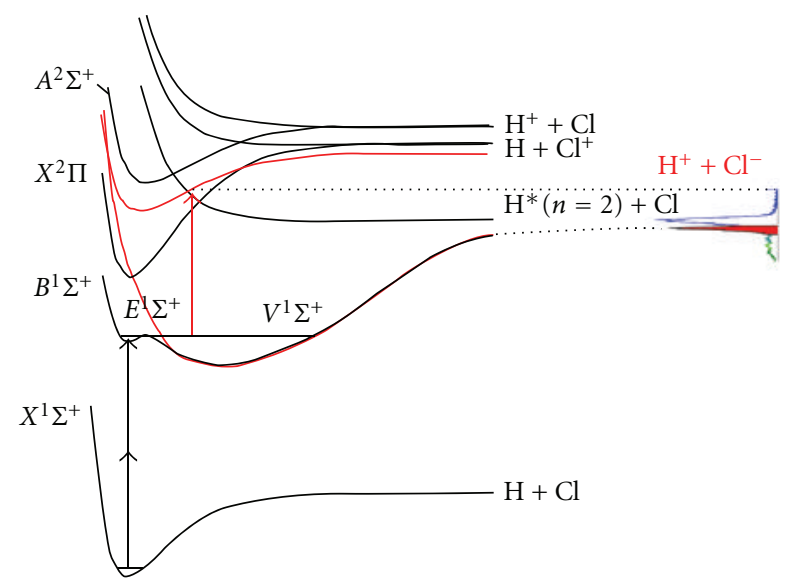

FIgURE 6: Path II: the medium fast observed $\mathrm{H}^{+}$ions are produced following a nonadiabatic transition from a bound Rydberg state to the ion-pair state at small internuclear distances. Here, only three photons are involved in the process altogether, the kinetic energy release being controlled in the third step. The colour coding matches that of Figure 3.

Recently, Kvaran and coworkers have detailedly studied Rydberg states of $\mathrm{HCl}$ with either $\Lambda>0$ or $S>0$ (i.e., belonging to the triplet manifold) or both, some of which exhibit near resonances with rovibrational levels of the $B^{1} \Sigma^{+}$ state [16-18]. The most prominent examples are the $Q(8)$ transition in the $F^{1} \Delta_{2} \leftarrow X^{1} \Sigma^{+}(1,0)$ band and the $Q(5)$ transition in the $f^{3} \Delta_{2} \leftarrow X^{1} \Sigma^{+}(0,0)$ band. Here, nearresonance interactions occur with single rotational levels in the $B^{1} \Sigma^{+}$state, namely, with $v^{\prime}=14, J^{\prime}=8$, and $v^{\prime}=8$, $J^{\prime}=5$, respectively. The energy difference between the levels is only $17.7 \mathrm{~cm}^{-1}$ for the triplet state and $11.3 \mathrm{~cm}^{-1}$ for the singlet state. Exciting those near-resonant levels resulted in a sharp increase of $\mathrm{Cl}^{+}$and $\mathrm{H}^{+}$ion signals. 


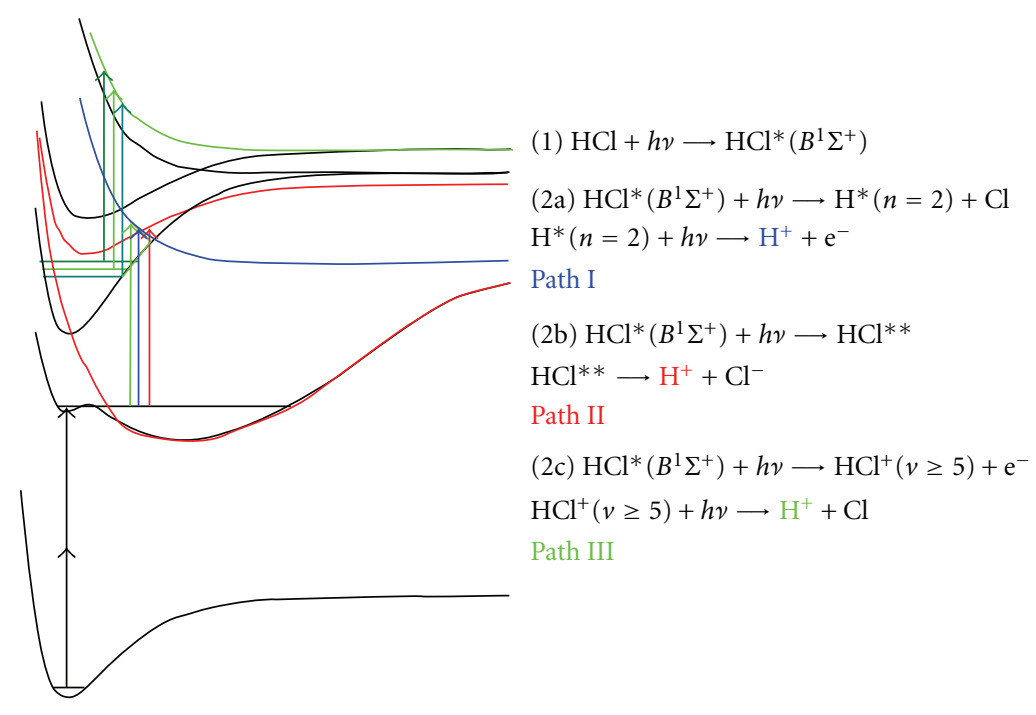

FIGURE 7: Illustration of the complex competition between fragmentation and ionization in the multiphoton excitation of $\mathrm{HCl}$ producing $\mathrm{H}^{+}$ions. Individual contributions shown in Figures 4 to 6 are combined into a common graph, the colour coding matches that of Figure 3 .

Kauczok et al. have recently studied the kinetic energy and the angular distributions of these fragments in order to identify those channels which become available to the $\mathrm{HCl}$ molecule in the near resonance situation [22]. They have found that those near-resonance rotational levels in the $f^{3} \Delta$ and $F^{1} \Delta$ states behave identically to the $B^{1} \Sigma^{+}$state levels.

However, subtle differences do exist, in particular for the excitation of the $f^{3} \Delta$ state. A small, but nonnegligible fragmentation yielding $\mathrm{H}^{+}$ions was observed for all rotational levels belonging to the $f^{3} \Delta$ state [18, 22]. It is believed to be due to the spin-allowed transition from the resonantly excited ${ }^{3} \Delta$ intermediate state to the ${ }^{3} \Pi$ gateway system leading to autoionization and dissociation as described in the previous section. Other than for $B^{1} \Sigma^{+}$excitation where the transition to the gateway state is believed to occur from the outer $(V)$ well of the $B$ state, the transition originating from the (single-well) $f^{3} \Delta$ state can only occur at the small internuclear distances associated with the inner $(E)$ well of the $B$ state. The observed angular anisotropies, however, suggest the participation of another fine structure component than the one with $\Omega=0$ which is accessed from $B^{1} \Sigma^{+}$. The equivalent background $\mathrm{H}^{+}$ion signal was not observed for excitation of the $F^{1} \Delta$ state, in agreement with the notion of a spin-forbidden transition to the gateway state which is neither facilitated by the peculiar shape of the double-well $B$ state nor by an allowed transition within the triplet manifold [22].

In addition, the strong $\mathrm{H}^{+}$ion signal enhancement observed for the near resonance $f^{3} \Delta_{2} Q(5)$ transition is associated with a slightly smaller kinetic energy release and a lowered anisotropy of the angular distribution compared to the adjacent rotational levels (cf. [22, Figure 3]), suggesting that the corresponding $\mathrm{Cl}$ partner fragment is formed in the excited ${ }^{2} P_{1 / 2}$ spin-orbit state. This behaviour contrasts the outcome of the dissociation via the ${ }^{3} \Pi$ gateway state which yields mainly ground state ${ }^{2} \mathrm{P}_{3 / 2} \mathrm{Cl}$ atoms as partner fragments $[3,19]$. This unexpected observation was explained by the lower photon energies used in ${ }^{3} \Delta_{2}$ excitation. While the near resonance with the $v^{\prime}=8, J^{\prime}=5$ level of the $B(V)$ state allows the excited $\mathrm{HCl}$ molecule to attain the large internuclear distance geometry of the outer $(V)$ well of the $B$ state, the Franck-Condon overlap with the above discussed ${ }^{3} \Pi_{0}$ gateway state has become small enough for a transition to another repulsive Rydberg state to dominate the transition to the former. Results from the observed angular anisotropies have not yet fully been understood which is why a final assignment of the gateway state could not be made.

\section{Angular Distributions of Photoproducts}

In general, the magnetic $M$ substrates of the two-photon excited state will not be equally populated after the twophoton transition. The nonequilibrium population of the magnetic substrates is equivalent to an excited state polarization (orientation and alignment) and can dramatically change the photoproduct angular distribution after the subsequent ionization/dissociation transitions.

The angular momentum polarization produced by twophoton absorption can be described by formalisms outlined by Manzhos et al. [1] and by Chichinin et al. [21]. In the axial recoil approximation the angular photoproduct distribution can be presented as a product of the excited state axis spatial distribution and the angular distribution of the photofragments produced via photolysis from an unpolarized excited state. This means that the influence of the excited state polarization on the photofragment angular distribution is independent of the particular multiphoton excitation mechanism. In other words, the two-photon excitation and the absorption of the third photon are independent, noncoherent processes.

Following the formalism outlined by Chichinin et al. [21] one obtains for the observed angular distribution of the photoproduct $P(\theta)$ :

$$
P(\theta)=P_{f}(\theta) P_{\mathrm{ph}}(\theta)=1+\sum \beta_{2 i} P_{2 i}(\cos \theta) .
$$


Here, $P_{f}(\theta)$ is the angular distribution of the axes in the intermediate state, and $P_{\mathrm{ph}}(\theta)$ is the photofragment angular distribution produced by the photolysis of the unpolarized intermediate state. For a one-photon photolysis process $P_{\mathrm{ph}}(\theta)$ can be characterized by a single anisotropy parameter $\beta_{\mathrm{ph}, 2}$ :

$$
P_{\mathrm{ph}}(\theta) \propto 1+\beta_{\mathrm{ph}, 2} P_{2}(\cos \theta) .
$$

Likewise, for the angular distribution of the axes in the intermediate state $P_{f}(\theta)$ one obtains

$$
P_{f}(\theta) \propto 1+\sum \beta_{f, 2 i} P_{2 i}(\cos \theta),
$$

where the upper limit for the summation depends on the excitation scheme as well as on the transition. However, often this expression is truncated after $i=1$, so that only the $\beta_{f, 2}$ parameter is considered, because higher-order terms are difficult to extract from experimental data owing to limitations by the signal to noise ratio.

Note however, that in the special case of the $Q(1)$ line $\left(J^{\prime \prime}=J^{\prime}=1\right)$ of the $B\left({ }^{1} \Sigma^{+}\right) \leftarrow X\left({ }^{1} \Sigma^{+}\right)$transition no terms higher than $\beta_{f, 2}$ exist, and that for the corresponding $Q(0)$ transition $\left(J^{\prime \prime}=J^{\prime}=0\right)$ no alignment in the two-photon excitation step can occur at all. In this case it is possible to first extract the value $\beta_{\mathrm{ph}, 2}$ from the observation of the $Q(0)$ transition and to subsequently obtain $\beta_{f, 2}$ from the corresponding analysis of the $Q(1)$ transition. Then, for $\beta_{2}$ one obtains

$$
\begin{gathered}
\beta_{2}=\beta_{\mathrm{ph}, 2} \quad \text { for } Q(0), \\
\beta_{2}=5 \frac{7\left(\beta_{\mathrm{ph}, 2}+\beta_{f, 2}\right)+2 \beta_{\mathrm{ph}, 2} \beta_{f, 2}}{35+7 \beta_{\mathrm{ph}, 2} \beta_{f, 2}} \text { for } Q(1) .
\end{gathered}
$$

Similar expressions might be derived for higher-order terms. Following this strategy Chichinin et al. obtained large and positive $\beta_{2}$ parameters for $\mathrm{H}^{+}$ions which decreased in the $Q(1)$ transition in comparison to the $Q(0)$ transition of the $B-X$ two-photon excitation [21].

The results are in agreement with excitation of the ${ }^{3} \Pi_{0}$ gateway state from the $B^{1} \Sigma^{+}$resonantly excited intermediate state in a parallel $\Delta \Omega=0$ transition characterized by a positive $\beta$ parameter.

The decrease of the $Q(1) \beta$ parameter compared to the $Q(0) \beta$ parameter is explained by the alignment of the excited $\mathrm{HCl} B^{1} \Sigma^{+}\left(J^{\prime}=1\right)$ molecule which was produced via the $Q(1)$ transition in a two-photon step. This alignment was necessarily absent for the $Q(0)$ transition, since the $J^{\prime}=0$ rotational state is isotropic. The observed alignment is due to the perpendicular nature of the two-photon step which occurs via the ${ }^{1} \Sigma \rightarrow{ }^{1} \Pi \rightarrow{ }^{1} \Sigma$ pathway, where the virtual one-photon intermediate state is the $A^{1} \Pi$ state. These results are in close agreement with a similar analysis performed by Manzhos et al. [1].

Thus, it is possible, by observing the angular distribution in a multiphoton ionization process, to identify the nature of the one-photon intermediate in two-photon excitation as well as that of the dissociative state.

\section{Conclusion and Outlook}

Although the photoionization of hydrogen chloride, a prototype of a heteronuclear diatomic molecule, has been intensively studied for decades, recently novel sophisticated experimental techniques have revealed several details associated with the competition of multiphoton ionization with fragmentation of $\mathrm{HCl}$. Chemical imaging techniques allow one to not only monitor the kinetic energy release of the ionized photofragments, but to observe the angular distribution of photoproducts simultaneously on an isotope and quantum state specific level.

Double-well potential energy curves like the one responsible for the peculiar fragmentation behaviour of $\mathrm{HCl}$ are likely to arise whenever two bound electronic states with minima at comparable energies, but significantly different nuclear geometries, form avoided crossings [41]. Consequently, double-well states have not only been observed in covalently bound closed shell molecules, such as hydrogen [42], the halogens [43, 44], and compounds thereof, but also in $\mathrm{NO}$ [45] and even in some van der Waals complexes $[46,47]$. In $\mathrm{HCl}$, the double minimum curve is formed from a ${ }^{1} \Sigma^{+}$Rydberg state correlating to $\mathrm{H}+\mathrm{Cl}^{*}$ neutral fragments and the ${ }^{1} \Sigma^{+}$ion-pair potential energy curve correlating to $\mathrm{H}^{+}+\mathrm{Cl}^{-}$fragments. Here, the small barrier between the inner and the outer well of the double minimum curve is of particular relevance, as the molecule either having surpassed the barrier or tunnelled through it, is subjected to a complex competition between ionization and fragmentation. This unusual behaviour of $\mathrm{HCl}$ is caused by subsequent photoabsorption processes of resonantly excited $\mathrm{HCl}$ at a very large internuclear distance.

Excitation schemes making use of the ${ }^{1} \Sigma^{+}$double minimum state produce $\mathrm{HCl}^{+}$parent molecular ions, electronically excited chlorine and electronically excited hydrogen atoms by absorption of three or four photons altogether in an energy range of 15 to $20 \mathrm{eV}$. Neutral and ionized hydrogen species are produced by several pathways. Accessing a common gateway state of ${ }^{3} \Pi_{0}$ symmetry, either autoionization of the $\mathrm{HCl}$ molecule into high vibrational levels of the electronic ground state of the molecular ion, followed by photodissociation of the vibrationally excited ion, or dissociation into a ground state chlorine atom and an electronically excited hydrogen atom takes place, the latter of which is easily ionized by the radiation field. A dissociation into the ion-pair $\mathrm{H}^{+}+\mathrm{Cl}^{-}$is also observed.

Currently, abundant experimental evidence is at hand which awaits thorough analysis on the basis of accurate high quality potential energy curves. Efforts are undertaken to provide such potentials without which the seemingly simple multiphoton interaction with a hydrogen containing diatomic molecule cannot fully be rationalized.

\section{References}

[1] S. Manzhos, C. Romanescu, H. P. Loock, and J. G. Underwood, "Two-photon state selection and angular momentum polarization probed by velocity map imaging: application to $\mathrm{H}$ atom photofragment angular distributions from the 
photodissociation of two-photon state selected $\mathrm{HCl}$ and $\mathrm{HBr}$," Journal of Chemical Physics, vol. 121, no. 23, pp. 11802-11809, 2004.

[2] C. Romanescu and H. P. Loock, "Photoelectron imaging following $2+1$ multiphoton excitation of $\mathrm{HBr}$," Physical Chemistry Chemical Physics, vol. 8, no. 25, pp. 2940-2949, 2006.

[3] C. Romanescu and H. P. Loock, "Proton formation in $2+1$ resonance enhanced multiphoton excitation of $\mathrm{HCl}$ and $\mathrm{HBr}$ via $(\Omega=0)$ Rydberg and ion-pair states," Journal of Chemical Physics, vol. 127, no. 12, Article ID 124304, 2007.

[4] H. P. Loock, B. L. G. Bakker, and D. H. Parker, "Photodissociation of superexcited states of hydrogen iodide: a photofragment imaging study using resonant multiphoton excitation at 13.39 and $15.59 \mathrm{eV}$," Canadian Journal of Physics, vol. 79, no. 2-3, pp. 211-227, 2001.

[5] M. Roth, C. Maul, K. H. Gericke, T. Senga, and M. Kawasaki, "State and energy characterisation of fluorine atoms in the A band photodissociation of $\mathrm{F}_{2}$," Chemical Physics Letters, vol. 305, no. 5-6, pp. 319-326, 1999.

[6] D. W. Chandler and P. L. Houston, "Two-dimensional imaging of state-selected photodissociation products detected by multiphoton ionization," The Journal of Chemical Physics, vol. 87, no. 2, pp. 1445-1447, 1986.

[7] D. H. Parker and A. T. J. B. Eppink, "Photoelectron and photofragment velocity map imaging of state-selected molecular oxygen dissociation/ionization dynamics," Journal of Chemical Physics, vol. 107, no. 7, pp. 2357-2362, 1997.

[8] A. T. J. B. Eppink and D. H. Parker, "Velocity map imaging of ions and electrons using electrostatic lenses: application in photoelectron and photofragment ion imaging of molecular oxygen," Review of Scientific Instruments, vol. 68, no. 9, pp. 3477-3484, 1997.

[9] A. I. Chichinin, T. Einfeld, C. Maul, and K. H. Gericke, "Threedimensional imaging technique for direct observation of the complete velocity distribution of state-selected photodissociation products," Review of Scientific Instruments, vol. 73, no. 4, p. 1856, 2002.

[10] A. I. Chichinin, T. Einfeld, K.-H. Gericke, and C. Maul, “3D imaging technique-observation of the three-dimensional product momentum distribution," in Imaging in Molecular Dynamics: Technology and Applications, B. Whitaker, Ed., Cambridge University Press, Cambridge, UK, 2003.

[11] S. Kauczok, N. Gödecke, A. I. Chichinin, M. Veckenstedt, C. Maul, and K. H. Gericke, "Three-dimensional velocity map imaging: setup and resolution improvement compared to three-dimensional ion imaging," Review of Scientific Instruments, vol. 80, no. 8, Article ID 083301, 2009.

[12] A. I. Chichinin, K. H. Gericke, S. Kauczok, and C. Maul, "Imaging chemical reactions-3D velocity mapping," International Reviews in Physical Chemistry, vol. 28, no. 4, pp. 607680, 2009.

[13] D. S. Green, G. A. Bickel, and S. C. Wallace, “ $(2+1)$ resonance enhanced multiphoton ionization of hydrogen chloride in a pulsed supersonic jet: spectroscopic survey," Journal of Molecular Spectroscopy, vol. 150, no. 2, pp. 303-353, 1991.

[14] D. S. Green, G. A. Bickel, and S. C. Wallace, " $(2+1)$ resonance enhanced multiphoton ionization of hydrogen chloride in a pulsed supersonic jet: spectroscopy and Rydberg $\sim$ valence interactions of the ${ }^{1} \Sigma^{+}\left(0^{+}\right)$and ${ }^{3} \Sigma^{-}\left(1,0^{+}\right)$states," Journal of Molecular Spectroscopy, vol. 150, no. 2, pp. 354-387, 1991.

[15] D. S. Green, G. A. Bickel, and S. C. Wallace, " $(2+1)$ resonance enhanced multiphoton ionization of hydrogen chloride in a pulsed supersonic jet: vacuum wavenumbers of rotational lines with detailed band analysis for excited electronic states of HCl," Journal of Molecular Spectroscopy, vol. 150, no. 2, pp. 388-469, 1991.

[16] Á. Kvaran, H. Wang, K. Matthiasson, A. Bodi, and E. Jónsson, "Two-dimensional $(2+\mathrm{n})$ resonance enhanced multiphoton ionization of $\mathrm{HCl}$ : photorupture channels via the $\mathrm{F}^{1} \Delta$ Rydberg state and ab initio spectra," Journal of Chemical Physics, vol. 129, no. 16, Article ID 164313, 2008.

[17] K. Matthíasson, H. Wang, and Á. Kvaran, “Two-dimensional $(2+n)$ REMPI of $\mathrm{HCl}$ : observation and characterisation of a new Rydberg state," Journal of Molecular Spectroscopy, vol. 255, no. 1, pp. 1-5, 2009.

[18] Á. Kvaran, K. Matthiasson, and H. Wang, "Two-dimensional $(2+n)$ resonance enhanced multiphoton ionization of $\mathrm{HCl}$ : state interactions and photorupture channels via low-energy triplet Rydberg states," Journal of Chemical Physics, vol. 131, no. 4, Article ID 044324, 2009.

[19] C. Romanescu, S. Manzhos, D. Boldovsky, J. Clarke, and H. P. Loock, "Superexcited state reconstruction of $\mathrm{HCl}$ using photoelectron and photoion imaging," Journal of Chemical Physics, vol. 120, no. 2, pp. 767-777, 2004.

[20] A. I. Chichinin, C. Maul, and K. H. Gericke, "Photoionization and photodissociation of $\mathrm{HCl}\left(\mathrm{B}^{1} \Sigma^{+}, \mathrm{J}=0\right)$ near 236 and 239 $\mathrm{nm}$ using three-dimensional ion imaging," Journal of Chemical Physics, vol. 124, no. 22, Article ID 224324, 2006.

[21] A. I. Chichinin, P. S. Shternin, N. Gödecke et al., "Intermediate state polarization in multiphoton ionization of $\mathrm{HCl}$," Journal of Chemical Physics, vol. 125, no. 3, Article ID 034310, 2006.

[22] S. Kauczok, C. Maul, A. I. Chichinin, and K. H. Gericke, "Proton formation dynamics in the REMPI[2+n] process via the $\mathrm{F}^{1} \Delta_{2}$ and $\mathrm{f}^{3} \Delta_{2}$ Rydberg states of $\mathrm{HCl}$ investigated by threedimensional velocity mapping," Journal of Chemical Physics, vol. 133, Article ID 024301, 2010.

[23] A. J. Yencha, D. Kaur, R. J. Donovan et al., "Ion-pair formation in the photodissociation of $\mathrm{HCl}$ and $\mathrm{DCl}$," The Journal of Chemical Physics, vol. 99, no. 7, pp. 4986-4992, 1993.

[24] J. B. Nee, M. Suto, and L. C. Lee, "Quantitative photoabsorption and fluorescence study of $\mathrm{HCl}$ in vacuum ultraviolet," The Journal of Chemical Physics, vol. 85, no. 2, pp. 719-724, 1986.

[25] P. Natalis, P. Pennetreau, L. Longton, and J. E. Collin, "Autoionization in $\mathrm{HCl}$ and $\mathrm{DCl}$ by photoelectron spectroscopy," Chemical Physics, vol. 73, no. 1-2, pp. 191-201, 1982.

[26] H. Frohlich and M. Glass-Maujean, "Photoabsorption, photodissociation, and photoionization cross sections of $\mathrm{HCl}$," Physical Review A, vol. 42, no. 3, pp. 1396-1404, 1990.

[27] H. Frohlich, P. M. Guyon, and M. Glass-Maujean, " $\mathrm{HCl}^{+}$ $\mathrm{X}$ vibrational states investigated from the $\mathrm{HCl}$ threshold photoelectron spectrum," Physical Review A, vol. 44, no. 3, pp. 1791-1795, 1991.

[28] E. de Beer, B. G. Koenders, M. P. Koopmans, and C. A. de Lange, "Multiphoton ionization processes in $\mathrm{HCl}$ studied by photoelectron spectroscopy," Journal of the Chemical Society, Faraday Transactions, vol. 86, no. 11, pp. 2035-2041, 1990.

[29] A. D. Pradhan, K. P. Kirby, and A. Dalgarno, "Theoretical study of $\mathrm{HCl}$ : potential curves, radiative lifetimes, and photodissociation cross sections," The Journal of Chemical Physics, vol. 95, no. 12, pp. 9009-9023, 1991.

[30] E. F. van Dishoeck, M. C. van Hemert, and A. Dalgarno, "Photodissociation processes in the $\mathrm{HCl}$ molecule," The Journal of Chemical Physics, vol. 77, no. 7, pp. 3693-3702, 1982.

[31] Y. F. Zhu, E. R. Grant, K. Wang, V. McKoy, and H. Lefebvre-Brion, "Spin-orbit autoionization and intensities in 
the double-resonant delayed pulsed-field threshold photoionization of HCl," The Journal of Chemical Physics, vol. 100, no. 12, pp. 8633-8640, 1994.

[32] H. Lefebvre-Brion and F. Keller, "Competition between autoionization and predissociation in the $\mathrm{HCl}$ and $\mathrm{DCl}$ molecules," The Journal of Chemical Physics, vol. 90, no. 12, pp. 7176-7183, 1989.

[33] P. J. Bruna and S. D. Peyerimhoff, "Excited-state potentials," in Ab Initio Methods in Quantum Chemistry, K. P. Lawley, Ed., John Wiley \& Sons, New York, NY, USA, 1987.

[34] R. Liyanage, R. J. Gordon, and R. W. Field, "Diabatic analysis of the electronic states of hydrogen chloride," Journal of Chemical Physics, vol. 109, no. 19, pp. 8374-8387, 1998.

[35] M. Bettendorff, S. D. Peyerimhoff, and R. J. Buenker, "Clarification of the assignment of the electronic spectrum of hydrogen chloride based on ab initio cl calculations," Chemical Physics, vol. 66, no. 3, pp. 261-279, 1982.

[36] L. Singleton and P. Brint, "Calculation of the Rydberg-state energies of HCl," Journal of the Chemical Society, Faraday Transactions, vol. 93, no. 1, pp. 21-23, 1997.

[37] Y. Li, O. Bludsky, G. Hirsch, and R. J. Buenker, "Ab initio configuration interaction study of the predissociation of the (4s), $(4 \mathrm{p} \sigma)^{1,3} \Pi$, and $(4 \mathrm{p} \pi)^{3} \Sigma^{+}$Rydberg states of $\mathrm{HCl}$ and DCl," Journal of Chemical Physics, vol. 112, no. 1, pp. 260-267, 2000.

[38] J. Pitarch-Ruiz, A. Sánchez de Merás, J. Sánchez-Marín, A. M. Velasco, C. Lavín, and I. Martín, "Low-lying Rydberg states of HCl," Journal of Physical Chemistry A, vol. 112, no. 14, pp. 3275-3280, 2008.

[39] D. Song, K. Liu, F. A. Kong, J. Li, and Y. Mo, "Ion-Pair dissociation dynamics of $\mathrm{HCl}$ : fast predissociation," Journal of Physical Chemistry A, vol. 113, no. 17, pp. 4919-4922, 2009.

[40] G. Vazquez and H. Lefebvre-Brion, private communication.

[41] H. Lefebvre-Brion and R. W. Field, The Spectra and Dynamics of Diatomic Molecules, Elsevier, Amsterdam, The Netherlands, 2004.

[42] E. R. Davidson, "First excited ${ }^{1} \Sigma_{\mathrm{g}}{ }^{+}$state of the hydrogen molecule," The Journal of Chemical Physics, vol. 35, pp. 11891202, 1961.

[43] P. J. Hay and D. C. Cartwright, "Rydberg, ionic, and valence interactions in the excited states of F," Chemical Physics Letters, vol. 41, no. 1, pp. 80-83, 1976.

[44] S. D. Peyerimhoff and R. J. Buenker, "Electronically excited and ionized states of the chlorine molecule," Chemical Physics, vol. 57, no. 3, pp. 279-296, 1981.

[45] R. de Vivie and S. D. Peyerimhoff, "Theoretical spectroscopy of the NO radical. I. Potential curves and lifetimes of excited states," The Journal of Chemical Physics, vol. 89, no. 5, pp. 3028-3043, 1988.

[46] M. Łukomski, J. Koperski, and M. Czajkowski, "Double-well potential energy curve of cadmium-krypton molecule in the B1 $\left(5^{3} \mathrm{P}_{1}\right)$ excited state," Spectrochimica Acta A, vol. 58, no. 8, pp. 1757-1767, 2002.

[47] E. Czuchaj, M. Kronicki, and H. Stoll, "Quasirelativistic valence ab initio calculation of the potential curves for the $\mathrm{Zn}$ rare gas van der Waals molecules," Chemical Physics, vol. 265, no. 3, pp. 291-299, 2001. 

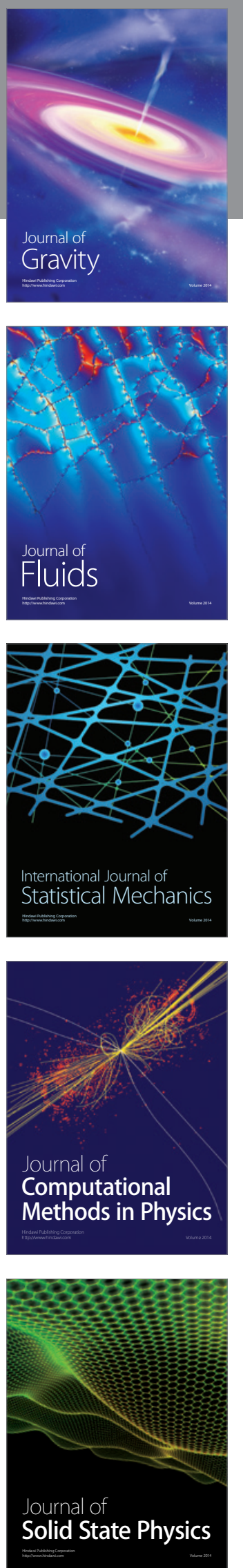

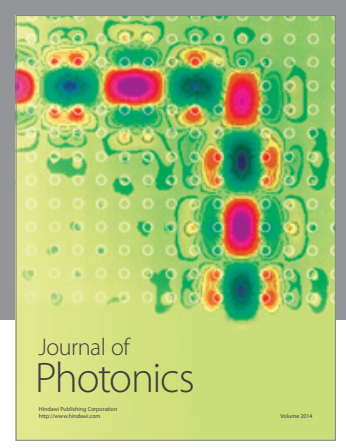

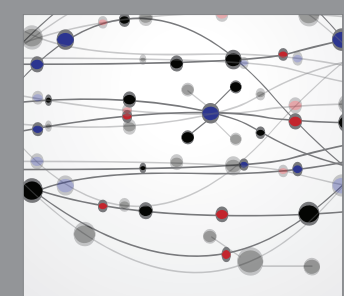

The Scientific World Journal
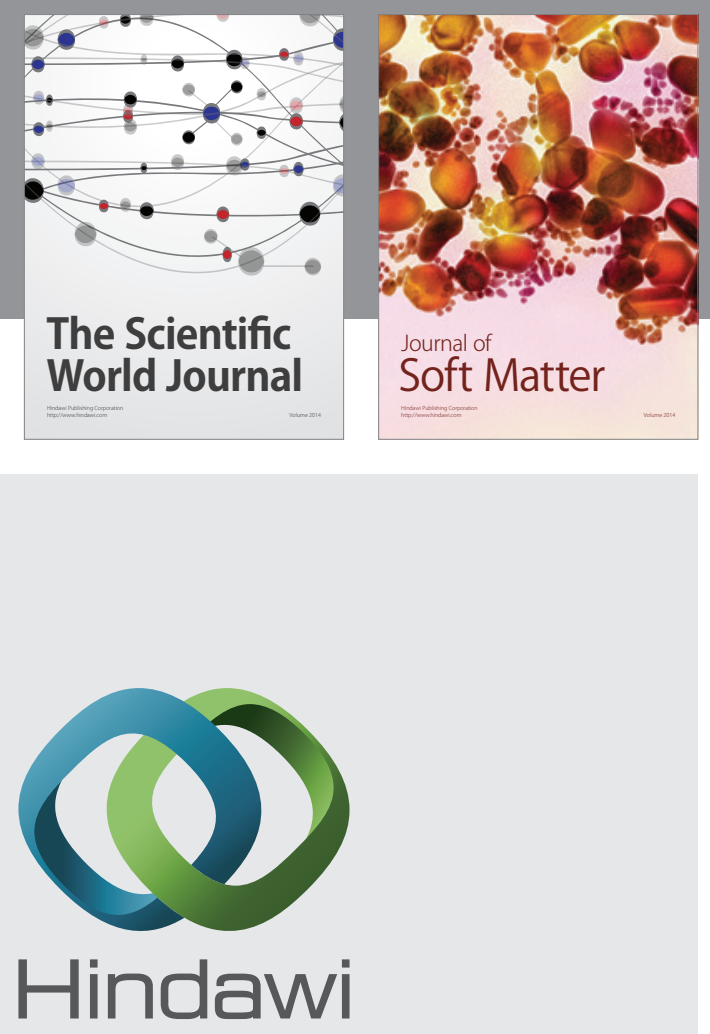

Submit your manuscripts at

http://www.hindawi.com
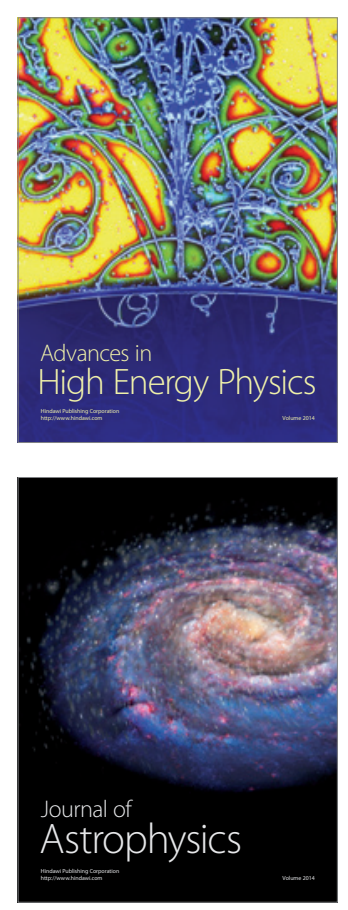
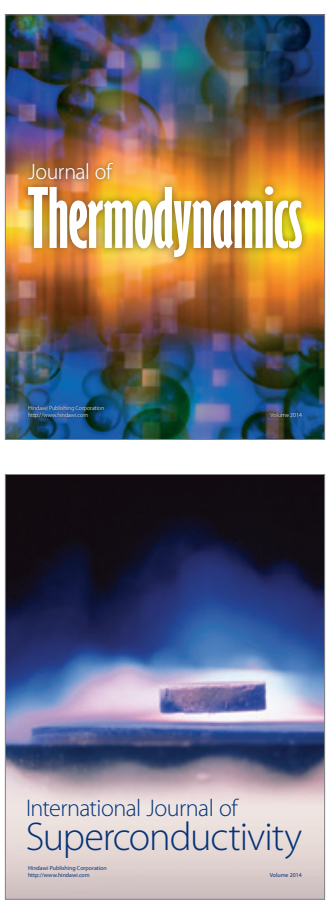
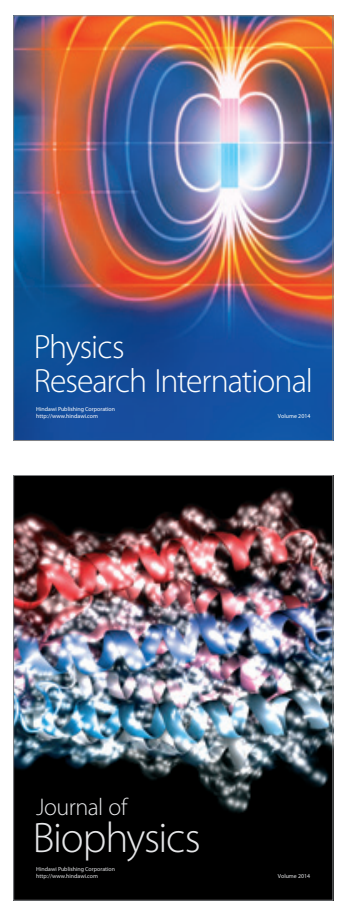
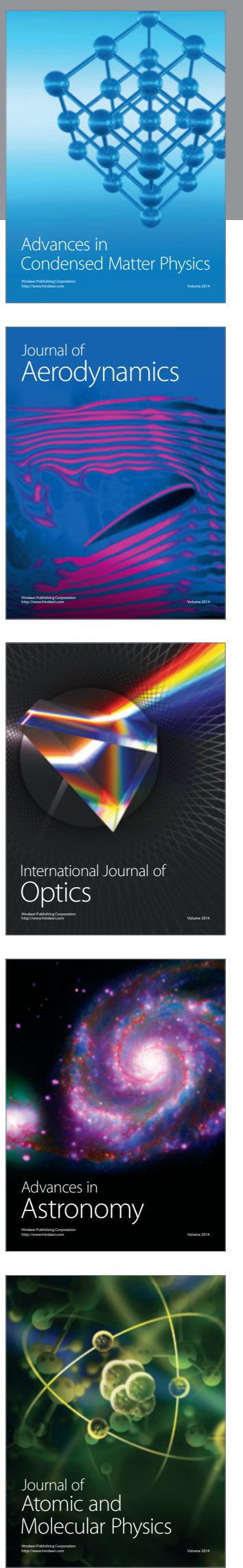